\title{
Correction to: Safety and efficacy of Cerebrolysin in acute brain injury and neurorecovery: CAPTAIN I-a randomized, placebo-controlled, double-blind, Asian-Pacific trial
}

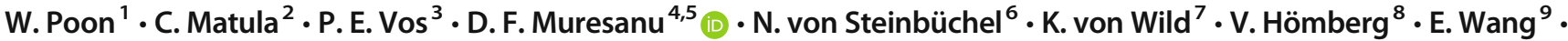 \\ T. M. C. Lee ${ }^{10} \cdot$ S. Strilciuc ${ }^{4,5} \cdot$ J. C. Vester ${ }^{11}$
}

Published online: 6 January 2020

(C) Fondazione Società Italiana di Neurologia 2020

\section{Correction to: Neurological Sciences (2019)}

https://doi.org/10.1007/s10072-019-04053-5

The above article was published online with incorrect abbreviations in Figures 2 and 3 last sentence of the legend. HDA should be corrected to HADS. The correct presentation has been corrected below:

"HDA-3LPCF, HADS: Anxiety Sumscore; HDD-3LPCF, HADS: Depression Sumscore; Visit No. 3 = Day 30".

The original article has been corrected.

The online version of the original article can be found at https://doi.org/ 10.1007/s10072-019-04053-5

D. F. Muresanu

dafinm@ssnn.ro

Division of Neurosurgery, Prince of Wales Hospital, The Chinese University of Hong Kong, Shatin, Hong Kong, China

2 Department of Neurosurgery, Medical University of Vienna, Vienna, Austria

3 Department of Neurology, Slingeland Hospital, Doetinchem, The Netherlands

4 Department of Clinical Neurosciences, "Iuliu Hatieganu" University of Medicine and Pharmacy, Cluj-Napoca, Romania

5 RoNeuro Institute for Neurological Research and Diagnostic, No. 37 Mircea Eliade Street, 400364 Cluj-Napoca, Romania
6 Institute of Medical Psychology and Medical Sociology, University Medical Centre Göttingen, Göttingen, Germany

7 Medical Faculty, Westphalia Wilhelm's University, Münster, Germany

8 Department of Neurology, SRH Gesundheitszentrum Bad Wimpfen GmbH, Bad Wimpfen, Germany

9 Department of Neurosurgery, National Neuroscience Institute, Singapore, Singapore

10 State Key Laboratory of Brain and Cognitive Sciences and Laboratory of Neuropsychology, The University of Hong Kong, Pokfulam, Hong Kong, China

11 Department of Biometry and Clinical Research, idv Data Analysis and Study Planning, Krailling, Germany 